\title{
$\mathbf{m} / \mathrm{S} 2000$
}

médecine/sciences $2000 ; 16: 1030-9$

\section{Les bases moléculaires de l'obésité : vers de nouvelles cibles thérapeutiques?}

\section{Liliane Michalik Béatrice Desvergne Walter Wahli}

L. Michalik, B. Desvergne, W. Wahli : Institut de biologie animale, Université de Lausanne, Bâtiment de biologie, CH-1015 Lausanne, Suisse.
Le nombre de sujets obèses dans les sociétés industrialisées est en constante augmentation, reflétant un environnement qui favorise les excès alimentaires et décourage l'exercice physique. Cependant, en dépit de ce contexte, pour la plupart des individus, l'organisme maintient le contrôle de son poids en ajustant l'équilibre énergétique. Chez les mammifères, les acteurs principaux des mécanismes de régulation de la balance énergétique sont la leptine, certains neuropeptides, les organes et les tissus impliqués dans le métabolisme des lipides et du glucose. Notre connaissance des mécanismes moléculaires participant au maintien de l'équilibre énergétique s'enrichit d'année en année, offrant un nombre croissant de cibles thérapeutiques potentiellement intéressantes pour le traitement de l'obésité.

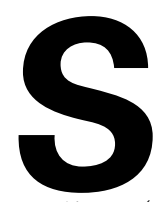

i l'obésité a été considérée autrefois comme un signe de prospérité, elle est à I'heure actuelle regardée comme une maladie à part entière $\left(m / s\right.$ 1996, $\left.n^{\circ} 12, p .1388\right)$. L'obésité est caractérisée par une surcharge pondérale importante due à une quantité excessive de lipides stockés dans le tissu adipeux blanc, et elle représente un facteur de risque déterminant dans le développement du diabète non-insulino-dépendant et des maladies cardio-vasculaires. Selon des données rassemblées par I'Organisation Mondiale de la Santé, I'obésité touche $10 \%$ des adultes en Europe de l'Ouest, $20 \%$ à $25 \%$ dans certaines régions des États-Unis et ce pourcentage peut atteindre jusqu'à $70 \%$ dans certaines populations. On peut actuellement parler d'épidémie en ce qui concerne l'obésité, et il existe un risque sérieux de voir se développer de façon dramatique les maladies qui lui sont associées.

Lorsqu'elle est bien adaptée, I'accumulation de triglycérides dans le tissu adipeux permet à l'organisme de mettre en réserve l'excédent d'énergie apporté par les nutriments en période d'abondance de nourriture (hydrates de carbone et lipides), de façon à pouvoir puiser dans ces réserves d'énergie en période de carence alimentaire. Aujourd'hui, les sociétés industrialisées nous permettent de disposer d'une nourriture abondante en permanence, et les périodes de jeûne sont devenues rares. Cependant, en termes d'évolution, cette situation est nouvelle et demeure limitée aux pays développés. L'homme, comme l'ensemble des mammifères, a donc acquis des mécanismes efficaces pour faire face au jeûne et lutter contre une perte de poids, aux dépens de mécanismes lui permettant de maîtriser une prise de poids excessive en situation de surabondance alimentaire. Nous sommes donc physiologiquement mieux armés pour nous adapter aux restrictions qu'aux excès de nourriture. Chez les rongeurs, plusieurs études ont démontré qu'une restriction alimentaire retardait le vieillissement. Ce retard se manifeste par une longévité augmentée, une pathologie réduite et un maintien des fonctions physiologiques dans un état plus juvénile. Le terrain génétique vient 
s'ajouter aux facteurs liés à notre environnement et à notre comportement. De nombreuses études familiales montrent en effet qu'il existe un fond héréditaire déterminant dans l'évolution du poids corporel. Cette notion de prédisposition génétique est désormais confirmée, puisque des mutations affectant certaines des voies métaboliques, qui seront décrites ci-dessous, ont été associées à l'apparition de l'obésité.

Le maintien d'un équilibre optimal entre la mise en réserve des lipides et leur utilisation nécessite donc des mécanismes physiologiques et moléculaires complexes. C'est le dérèglement de cet équilibre, fréquemment dû à l'accumulation des facteurs déjà mentionnés (génétiques, environnementaux, nutritionnels), qui conduit à l'obésité. Les éléments participant au contrôle de cet équilibre peuvent être regroupés en trois catégories principales.

Il existe tout d'abord des mécanismes visant à informer l'organisme sur la quantité de lipides stockés dans le tissu adipeux, lui permettant ainsi de réagir de manière appropriée, soit en poursuivant la mise en réserve, soit en utilisant ces réserves. L'un de ces mécanismes est la sécrétion de leptine par le tissu adipeux lui-même, mais il est vraisemblable que d'autres signaux existent. Le taux de leptine sécrétée doit ensuite être enregistré pour être transmis aux organes effecteurs. Ce signal est intégré au niveau du système nerveux central, et plus particulièrement de I'hypothalamus (pour revue, voir [1]). Finalement, la transmission du signal engendre une réponse adaptative de la part de nombreux organes et tissus. Cette réponse consiste $d^{\prime}$ une part à sélectionner un comportement approprié vis-à-vis de la prise de nourriture, et d'autre part à augmenter ou à utiliser les réserves en lipides, suivant les besoins.

Dans cet article, nous avons choisi de décrire les acteurs périphériques participant au maintien de l'équilibre énergétique. En particulier, nous insisterons sur les fonctions des facteurs de transcription PPAR (peroxisome proliferator activated-receptors). En effet, les PPAR sont fortement impliqués dans la régulation du métabolisme des lipides, et sont en outre des cibles dans le traitement de maladies associées à l'obésité.

\section{Les molécules " signal "} La leptine

La leptine, hormone produite principalement par les adipocytes du tissu adipeux blanc, est un élément important de la régulation du métabolisme des lipides et du glucose (pour revue, voir [2]). Chez la souris, plusieurs mutations affectant le fonctionnement de cette hormone ont été identifiées. L'une touche le gène de la leptine (gène $o b$ ) [3], tandis que l'autre affecte le gène codant pour le récepteur hypothalamique (gène $d b$ ) [4] de cette hormone. Ces animaux ont un comportement hyperphagique, souffrent d'obésité, développent une insulino-résistance sévère, présentent des défauts de croissance et de fertilité, ainsi qu'une dépense énergétique réduite. Bien que rares chez I'homme, des mutations du gène codant pour la leptine $[5,6]$ ou son récepteur [7] ont également été découvertes chez des patients souffrant d'obésité. Ces patients ont un comportement hyperphagique, mais ne développent pas de résistance sévère à l'insuline. Leur puberté est généralement retardée de façon importante, suggérant que le rôle de la leptine n'est pas limité au contrôle de la balance énergétique chez I'homme.

La leptine est fréquemment considérée comme une hormone permettant de diminuer la masse du tissu adipeux. Elle agit sur la prise alimentaire à la fois grâce à ses propriétés anorexigènes, et/ou en augmentant la dépense énergétique. Cependant, il est possible que l'action principale de la leptine ne soit pas de diminuer la masse adipeuse, mais qu'elle représente plutôt un signal permettant de préserver un stock suffisant d'énergie sous forme de graisse [8]. La quantité de leptine circulante, corrélée à la masse de tissu adipeux, est le reflet de l'état de ce stock. Or, la variation de la quantité relative de leptine produite par les cellules adipeuses est beaucoup plus sensible à la baisse qu'à l'augmentation de poids. Une diminution importante de la leptinémie lors d'une perte de poids signalerait donc une réduction des réserves d'énergie, et aurait pour conséquence une adaptation de l'organisme, en vue d'un retour à des quantités suffisantes de réserves: hyperphagie, stockage préférentiel des calories sous forme de graisse et, chez la souris, baisse de la dépense énergétique. Comme nous I'avons souligné plus haut, le développement de mécanismes permettant de s'adapter aux périodes de jeûne confère un avantage évident en termes d'évolution. A l'inverse, les variations minimes de la leptinémie en période d'abondance traduirait le manque de mécanismes permettant de lutter contre une prise de poids exagérée. Bien entendu, le seuil de leptine circulante susceptible de signaler à l'organisme, au niveau du système nerveux central, que les réserves de tissu adipeux sont suffisantes varie en fonction de chaque individu, et dépend probablement de facteurs génétiques. Si ce modèle s'avère exact, I'utilisation de la leptine dans un but thérapeutique viserait non pas à provoquer la perte de poids, mais à maintenir la quantité de leptine au niveau de ce seuil, de façon à ne pas déclencher les mécanismes favorisant la mise en réserve de graisse (m/s 1999, $\left.n^{\circ} 11, p .1276\right)$.

\section{Un nouveau candidat: \\ le facteur FIAF}

Le FIAF (fasting induced adipose factor), également décrit sous le nom de PGAR (PPAR $\gamma$ angiopoietin related) ou HFARP (hepatic fibrinogen/angiopoietin-related protein), a été identifié très récemment, chez I'homme et la souris [9-11]. Cette glycoprotéine, apparentée au fibrinogène et aux angiopoïétines, est produite essentiellement par les tissus adipeux blanc et brun, le placenta, et en quantité moindre par le foie. Il s'agit d'une protéine sécrétée, et sa présence dans le plasma suggère qu'elle pourrait avoir une fonction endocrine. Le gène codant pour cette protéine est une cible de PPAR $\alpha$ dans le foie [11], et de PPAR $\gamma$ dans des adipocytes en culture [10], et son expression est contrôlée positivement par ces deux isotypes. L'expression du transcrit de FIAF est augmentée dans le foie de souris nourries avec un activateur de PPAR $\alpha$ [11], ainsi que dans des cultures d'adipocytes traitées par un activateur de PPAR $\gamma$ [10]. A I'inverse, chez les souris PPAR $\alpha^{-1}$, ou $P P A R \gamma^{+-}$, le transcrit de FIAF est moins exprimé respectivement dans le foie et 
dans le tissu adipeux blanc, [11]. La quantité de FIAF présente dans le plasma augmente en période de jeûne, et décroît lorsque les animaux sont soumis à un régime riche en graisses [11]. Ce résultat est renforcé par I'observation d'une augmentation de l'expression de FIAF dans le tissu adipeux blanc chez des souris soumises à une période de jeûne, le taux de départ étant rétabli après un apport de nourriture. En outre, dans les tissus adipeux blanc et brun, le transcrit de FIAF est présent en quantité deux à trois fois supérieure chez des souris obèses ob/ob ou db/db [10].

La fonction de la protéine FIAF est encore inconnue. Cependant, chez la souris, sa présence dans le plasma, ainsi que la régulation de son expression en fonction de l'état nutritionnel suggèrent fortement que cette protéine pourrait participer aux mécanismes de maintien de l'équilibre énergétique. La régulation de son expression étant inverse de celle de la leptine, il est également tentant d'imaginer que FIAF pourrait avoir une fonction endocrine complémentaire de celle de la leptine. Cette hypothèse n'est pas vérifiée à ce jour et, en particulier, I'existence d'un récepteur et de tissus cibles de FIAF restent à démontrer.

\section{Les acides gras}

Parmi les autres molécules «signal» figurent très vraisemblablement les acides gras eux-mêmes. Ceux-ci sont capables d'interagir directement avec les PPAR et de moduler l'activité transcriptionnelle de ces récepteurs nucléaires. De nombreux arguments, obtenus par des expériences in vitro mais également in vivo, plaident en faveur du fait que les acides gras contribuent à informer l'organisme sur son état nutritionnel, et participent de cette façon à la régulation de la balance énergétique (pour revue, voir [12]). Ainsi, les acides gras ne sont pas seulement des molécules énergétiques passives, mais se comportent également comme des "signaux» interagissant directement avec les PPAR.

\section{Les organes et tissus effecteurs de l'équilibre énergétique: importance des PPAR}

Les organes ou les tissus effecteurs impliqués dans le maintien de l'équilibre énergétique sont principalement les tissus adipeux blanc et brun, le foie, le muscle squelettique et le pancréas. Les mécanismes par lesquels les signaux provenant du système nerveux central sont transmis à ces organes périphériques sont encore loin d'être élucidés. Ces organes effecteurs sont aussi capables de se transmettre des signaux et de communiquer directement, sans que le système nerveux central ne soit nécessairement impliqué. Ce chapitre est surtout consacré aux récepteurs nucléaires PPAR, qui sont au centre des mécanismes permettant de régler l'équilibre énergétique, et en particulier le métabolisme des lipides, au niveau des organes et tissus périphériques.

Tableau I. Mutations associées au développement de l'obésité chez I'homme*.

Gène muté $\mathbf{N}$ sujets

Mutation

Conséquences pathologiques

\begin{tabular}{|c|c|c|c|}
\hline \multirow{3}{*}{ Leptine [6] } & 3 & Substitution $1 \mathrm{NT}$ & Obésité, hyperphagie \\
\hline & & (C-T; codon 105 du gène de la leptine), & \\
\hline & 2 & $\begin{array}{l}\text { substitution d'une arginine par un tryptophane } \\
\text { Délétion } 1 \mathrm{NT} \text { (G : codon } 133 \text { du gène de la }\end{array}$ & $\begin{array}{l}\text { Taux de leptine circulante très faible } \\
\text { Obésité hvperphagie }\end{array}$ \\
\hline \multirow{2}{*}{ Leptine [5] } & & leptine), décalage du cadre de lecture & Taux de leptine circulante très faible \\
\hline & & à partir de la glycine 132 , protéine tronquée & \\
\hline \multirow{3}{*}{$\begin{array}{l}\text { Récepteur } \\
\text { de la leptine } \\
\text { [7] }\end{array}$} & 3 & Substitution 1 NT (G-A; site d'épissage de & Obésité, hyperphagie \\
\hline & & l'exon 16 du gène du récepteur à la leptine); & Taux élevé de leptine circulante \\
\hline & & protéine tronquée, dépourvue des régions & \\
\hline PPAR $\gamma 2$ [23] & 4 & $\begin{array}{l}\text { transmembranaire et intracellulaire } \\
\text { Substitution } 1 \text { NT (G-T), substitution } 1 \text { aa } \\
\text { (Proline } 115 \text { Glutamine) }\end{array}$ & Obésité, diabète insulinorésistant (3/4) \\
\hline PPAR $\gamma 2 *$ [24] & 329 & $\begin{array}{l}\text { Gain d'activité de la protéine } \\
\text { Substitution } 1 \mathrm{NT}^{* *}(\mathrm{G}-\mathrm{C}) \text {, substitution } 1 \text { aa } \\
\text { (Proline } 12 \text { Alanine) }\end{array}$ & $\begin{array}{l}\text { Pas d'obésité, } \\
\text { Indice de masse corporelle diminué, }\end{array}$ \\
\hline \multirow[t]{5}{*}{ PPAR $\gamma 2$ [46] } & 3 & $\begin{array}{l}\text { Perte d'activité de la protéine } \\
\text { Substitution } 1 \text { NT }(C-T) \text {, substitution } 1 \text { aa }\end{array}$ & $\begin{array}{l}\text { Amélioration de la sensibilité à l'insuline } \\
\text { Pas d'obésité, diabète insulinorésistant }\end{array}$ \\
\hline & & (Proline 467 Leucine) 2/3 & \\
\hline & & Substitution $1 \mathrm{NT}$ (G-A), substitution 1 aa & \\
\hline & & (Valine 290 Méthionine) 1/3 & \\
\hline & & $\begin{array}{l}\text { Diminution d'activité de la protéine, } \\
\text { associée à une activité dominant négatif }\end{array}$ & \\
\hline
\end{tabular}

Diverses mutations touchant des gènes codant pour des acteurs clés de la régulation du métabolisme des lipides ont été identifiées. Dans la plupart des cas, ces mutations sont récessives, comme le montre l'étude des antécédents familiaux. Bien que rares (la fréquence de mutations homozygotes est probablement $<<10^{-3}$ ), ces mutations apportent des informations importantes sur la régulation du poids corporel chez l'homme.

Á l'exception de PPAR 2 , pour lequel deux types de mutations ne sont pas associées au développement de l'obésité, tous les sujets identifiés sont caractérisés par une apparition précoce du syndrome (quelques mois après la naissance). Dans certains cas, le syndrome d'obésité est également associé à des troubles du comportement, ainsi qu'à une croissance et une puberté retardée.

$N$ sujets: nombre de sujets chez lesquels la mutation a été identifiée; NT: nucléotide; aa: acide aminé.

*: Ce tableau regroupe les éléments traités dans cette revue et cette liste n'est pas exhaustive.

** : Plutôt qu'une mutation, cette substitution peut être considérée comme un polymorphisme. 


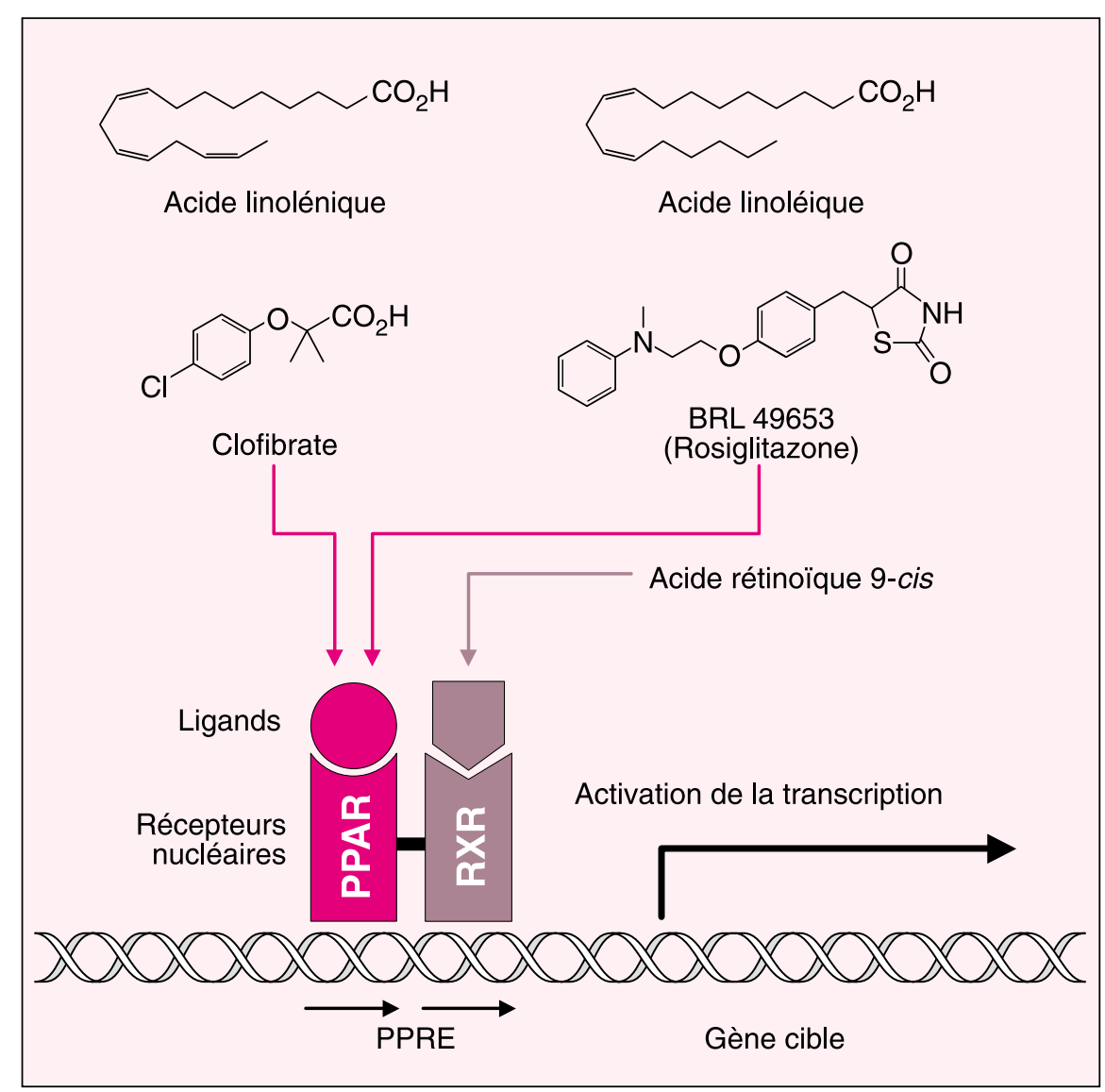

Figure 1. Les PPAR: récepteurs nucléaires activés par un ligand. Les PPAR sont des facteurs de transcription dont l'activité dépend de l'interaction avec un ligand. Parmi ces derniers, les acides gras tels que l'acide linoléique ou I'acide linolénique lient les trois PPAR, avec cependant une préférence pour $P P A R \alpha$. Le clofibrate est un ligand de PPAR $\alpha$ utilisé pour ses propriétés hypolipidémiantes. La rosiglitazone, ou BRL, appartient à la famille des thiazolidinediones, antidiabétiques ligands de PPAR . Après leur liaison avec un ligand, les PPAR, sous forme d'hétérodimères avec leur partenaire $R X R$, interagissent avec des séquences spécifiques appelées PPRE (PPAR response element) présent dans le promoteur de leurs gènes cibles, et activent alors la transcription de ces gènes. L'acide rétinoïque 9-cis est le ligand de $R X R$, qui peut également participer à l'activation du complexe PPAR-RXR.

\section{Les PPAR}

Les PPAR forment une famille de trois récepteurs, PPAR $\alpha, \beta(\delta)$ et $\gamma$ (respectivement NR1C1, NR1C2 et NR1C3) [13], qui appartiennent à la classe des récepteurs nucléaires dont l'activité est stimulée par un ligand (pour revue, voir [12]). Les PPAR se lient à des régions promotrices spécifiques de leurs gènes cibles sous forme d'hétérodimères avec leur partenaire RXR. En réponse à leur liaison avec un ligand, ils activent la transcription de ces gènes (figure 1). L'isotype plus grande affinité. Les eicosanoides 8(S)HETE et LTB4 sont également des ligands de PPAR $\alpha$, ainsi que les fibrates utilisés en clinique comme molécules hypolipidémiantes. La 15désoxy D-prostaglandine J2 est également un ligand naturel de PPAR $\gamma$, ainsi que les antidiabétiques de la classe des thiazolidinediones (TZD) (figure 1) (pour revue, voir [14]).

$\mathrm{Si}$ les fonctions biologiques de PPAR $\beta$ dans le contrôle de la balance énergétique sont encore peu connues à I'heure actuelle, de nombreuses observations permettent de souligner les rôles opposés et complémentaires de PPAR $\alpha$ et PPAR $\gamma$ dans la régulation du métabolisme lipidique (figure 2). PPAR $\alpha$, dont la fonction est étudiée essentiellement dans les hépatocytes, contrôle un ensemble de gènes impliqués dans diverses étapes du catabolisme des lipides, telles que le transport des acides gras à travers les membranes, leur transport intracytoplasmique, et leur oxydation microsomale, peroxisomale et mitochondriale (pour revue, voir [12]). PPAR $\gamma$, à l'inverse de PPAR $\alpha$, contrôle positivement des gènes impliqués dans la lipogenèse et fait partie intégrante du processus de différenciation adipocytaire [15]. Plus récemment, PPAR $\beta$ est apparu comme un acteur potentiel de la cascade de différenciation adipocytaire [16].

\section{Le stockage des triglycérides dans le tissu adipeux blanc et la différenciation adipocytaire: rôle de PPAR $\gamma$}

Lorsque la quantité moyenne d'énergie absorbée est supérieure à la quantité moyenne d'énergie consommée, l'excédent est mis en réserve dans le tissu adipeux blanc (triglycérides) et le foie (glycogène). L'excès de stockage sous forme de lipides dans le tissu adipeux blanc, caractéristique de l'obésité, fait intervenir à la fois une augmentation de la taille (hypertrophie) et du nombre (hyperplasie) des adipocytes. PPAR $\gamma$ est un élément-clé dans ce processus, qui régit à la fois la différenciation du tissu adipeux blanc et la lipogenèse.

Deux observations ont initialement suggéré le rôle de PPAR $\gamma$ dans la différenciation des adipocytes: la première est l'expression élevée de ce 


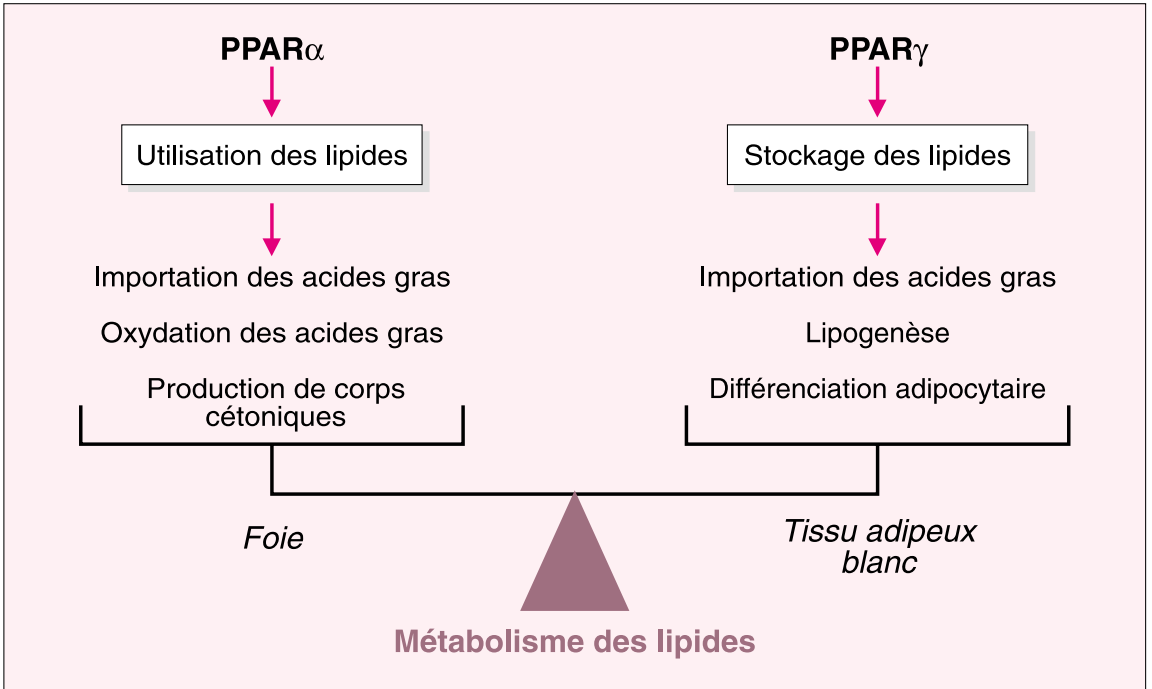

Figure 2. PPAR $\alpha$ et PPAR $\gamma$ : deux acteurs aux fonctions opposées et complémentaires dans la régulation du métabolisme des lipides. L'étude des gènes cibles de PPAR $\alpha$ et PPAR $\gamma$ donne une vue d'ensemble de leurs fonctions respectives. Ces deux récepteurs sont des facteurs essentiels de la régulation du métabolisme lipidique. L'activité de PPAR $\alpha$ vise à l'utilisation des acides gras comme source d'énergie, tandis que celle de PPARy est destinée à la mise en réserve des lipides sous forme de triglycérides. Le rôle de PPAR $\beta$ demeure obscur.

récepteur dans le tissu adipeux blanc, et la deuxième est l'augmentation de I'expression de PPAR $\gamma$ dans des lignées cellulaires pré-adipocytaires, qui précède celle de marqueurs de différenciation adipocytaires [17]. Le mécanisme moléculaire de l'activité adipogénique de PPAR $\gamma$ fait intervenir le cofacteur PGC-2. Celui-ci, en interagissant avec la région $\mathrm{N}$-terminale de PPAR $\gamma$, stimule son activité adipogénique dans une lignée de pré-adipocytes [18]. In vivo, l'expression de PPAR $\gamma$ est contrôlée, entre autres, par la quantité de lipides présents dans le régime alimentaire. Chez le rat et la souris, elle augmente de façon significative dans le tissu adipeux blanc lorsque le régime alimentaire est riche en lipides, et augmente également dans le foie chez des souris obèses $[19,20]$. A l'inverse, elle est réduite après un jeûne de 48 heures [21]. L'expression de PPAR $\gamma$ a également été étudiée chez l'homme. Chez des sujets obèses, l'expression de PPAR 2 est plus importante dans le tissu adipeux blanc [22], et un régime alimentaire pauvre en calories abaisse l'expression de PPARy2 dans ce tissu, suggérant que cette isoforme est particulièrement impliquée dans le contrôle de la fonction adipocytaire. Ces résultats soulignent le rôle clé de PPAR $\gamma$ dans la stimulation du stockage de lipides dans le tissu adipeux blanc, et vont de pair avec les conséquences des mutations de ce gène observées dans certaines populations. Deux types de mutations, touchant le gène de PPAR $\gamma$ et affectant I'indice de masse corporel, ont en effet été identifiées (tableau I). La première a été identifiée chez des patients souffrant d'obésité sévère [23], et entraîne la substitution d'une proline en glutamine en position 115 de la protéine, qui induit une augmentation de l'activité de PPAR $\gamma$. La seconde mutation, qui peut être considérée comme un polymorphisme en raison de sa fréquence, entraine la substitution d'une proline en alanine à la position 12 de la protéine et une diminution de l'activité de PPARr. A l'inverse de la précédente, cette mutation serait associée à un indice de masse corporel plus faible dans la population concernée [24].

Les adipocytes produisent de la leptine, proportionnellement à la quantité de triglycérides stockés, et cette production par le tissu adipeux blanc est contrôlée négativement par PPAR $\gamma$ [25]. L'analyse de souris portant une délétion du gène de PPAR à l'état

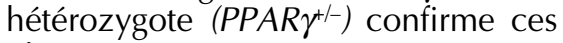
observations, puisque ces animaux présentent une augmentation de la quantité de leptine circulante [26]. Cette régulation laisse penser qu'il existe une corrélation inverse entre le taux d'expression de PPAR $\gamma$ et celui de leptine sécrétée. Paradoxalement, les observations faites à ce jour ne confirment pas cette hypthèse, puisque l'état de réplétion adipeuse se traduit par un taux élevé de leptine et une augmentation de l'expression de PPAR $\gamma$, alors que le jeûne entraîne une chute de ces deux paramètres $[20,27]$. Ces résultats montrent bien la complexité des interconnexions hormonales et le chemin qu'il nous reste à parcourir pour mieux les comprendre.

\section{La différenciation adipocytaire: rôle de PPAR $\beta$}

Les fonctions de PPAR $\beta$ sont encore peu connues. Cependant, ce récepteur est exprimé en quantité significative dans le tissu adipeux blanc, et son expression augmente au cours des étapes initiales de la différenciation de pré-adipocytes en culture [28]. Son expression ectopique dans une lignée de fibroblastes murins suggère que PPAR $\beta$ pourrait être un acteur précoce du processus de différenciation adipocytaire. Dans ce modèle, la différenciation des cellules en adipocytes n'est obtenue que si des activateurs de PPAR $\beta$ et PPAR $\gamma$ sont présents. L'activation de PPAR $\beta$ par des acides gras à longue chaine conduit à une augmentation de l'expression de PPAR $\gamma$, dont l'activité serait alors nécessaire à la différenciation finale de ces fibroblastes en adipocytes [16]. Il est dès lors tentant de penser que PPAR $\beta$ pourrait être impliqué dans l'augmentation du nombre de cellules adipeuses observée sous l'effet d'un régime riche en graisses. Chez la souris, I'ablation des deux allèles du gène codant pour PPAR $\beta$ semble induire une légère diminution de la masse adipeuse. PPAR $\beta$ pourrait donc jouer un rôle dans le développement du tissu adipeux blanc in vivo, sans toutefois être indispensable [29]. 


\section{Le catabolisme des lipides dans le foie: rôle de PPAR $\alpha$}

De manière générale, PPAR $\alpha$ est exprimé parallèlement aux activités de $\beta$-oxydation peroxisomale et mitochondriale des acides gras, en particulier dans des tissus utilisant les acides gras comme source d'énergie principale.

L'oxydation des acides gras (catabolisme) contribue à une diminution des réserves de triglycérides, et PPAR $\alpha$ contrôle directement cette voie, puisque dans le foie, ce récepteur exerce un contrôle positif sur le transport des acides gras dans les hépatocytes, leur activation en acyl-CoA et leur oxidation (figure 2). En période de jeûne, la lipolyse est stimulée dans le tissu adipeux blanc, ce qui conduit à une augmentation du taux d'acides gras circulants, prélevés et dégradés par les hépatocytes. Leur oxydation conduit à la production de corps cétoniques tout en permettant parallèlement la néoglucogenèse. L'expression de PPAR $\alpha$ est augmentée dans le foie par l'action des glucocorticoïdes, dont les taux sont élevés pendant le jeûne. De plus, les acides gras sont des ligands des PPAR, avec une affinité particulièrement élevée pour PPAR $\alpha$. II est donc probable que, lors d'une libération importante d'acides gras en période de jeûne, les acides gras eux-mêmes participent à la stimulation de leur propre catabolisme en activant PPAR $\alpha$, mais cette hypothèse demeure lacunaire (pour revue, voir [12]).

L'étude du phénotype de souris déficientes en PPAR $\alpha\left(P P A R \alpha^{1-}\right)$ a également contribué à démontrer l'implication de PPAR $\alpha$ dans la régulation de I'équilibre énergétique. Lorsqu'elles sont nourries, la déficience en PPAR $\alpha$ n'a pas de conséquences directement observables chez ces souris. En revanche, lors d'un jeûne, les processus d'oxydation des acides gras et de cétogenèse sont défectueux, et les conséquences observées sont multiples: taux d'acides gras circulants élevé et accumulation de lipides dans le foie, hypothermie, hypoglycémie et hypocétonémie [30, 31]. Ce phénotype souligne le rôle crucial de PPAR $\alpha$ dans I'utilisation et la production de molécules énergétiques en situation de jeûne.
Il est d'autre part important de noter que les premiers composés réellement efficaces dans le traitement de I'hyperlipidémie ont été les fibrates. Ces hypolipidémiants, qui sont des ligands de PPAR $\alpha$, ont pour effet de stimuler le catabolisme des lipides et de réduire la production des VLDL, particules de transport des triglycérides.

\section{La thermogenèse adaptative: rôles de PPAR $\alpha$ et PPAR $\gamma$}

La dépense énergétique est l'un des facteurs importants dans le maintien du poids corporel, et la thermogenèse adaptative est une façon efficace de dépenser de l'énergie. Elle consiste en la production de chaleur, en réponse à des facteurs environnementaux, tels que le régime alimentaire (jeûne ou abondance) ou la température ambiante. Elle représente une protection efficace contre le froid, qui permet également de brûler d'éventuelles calories excédentaires. Dans ce contexte, le tissu adipeux brun, dont les adipocytes particulièrement riches en mitochondries sont distincts des adipocytes blancs sur le plan morphologique et fonctionnel, joue un rôle essentiel. La thermogenèse est possible dans les adipocytes bruns grâce à la présence d'une protéine mitochondriale, I'UCP1 (uncoupling protein 1), capable de découpler I'oxydation des acides gras de la phosphorylation de I'ATP, et d'aboutir à une libération d'énergie sous forme de chaleur. C'est ainsi que des souris déficientes en UCP1 règlent mal leur température lors d'une exposition au froid, mais ne développent pas d'obésité [32]. Cela suggère que le rôle d'UCP1 dans la régulation de la température corporelle est essentiel, ainsi que, de manière surprenante, sa faible importance dans le maintien du poids corporel. Trois protéines apparentées à UCP1 ont été identifiées: UCP2, dont le profil d'expression est très large, UCP3, exprimée dans le muscle squelettique et le tissu adipeux brun murin et UCP4 exprimée exclusivement dans le cerveau [33, 34].

Le contrôle transcriptionnel de I'expression des UCP fait intervenir les PPAR. PPAR $\gamma$ intervient dans la régulation positive de l'expression
d'UCP1 [35], et cette activité est fortement stimulée par la présence de PGC-1. PGC-1 est un coactivateur exprimé principalement dans le tissu adipeux brun, le cœur, le rein, le cerveau et le muscle squelettique. L'absence de PGC-1 dans le tissu adipeux blanc, ainsi que l'induction rapide de son expression dans le tissu adipeux brun et le muscle chez des souris exposées au froid, suggèrent un lien important entre ce cofacteur, I'activité de PPAR $\gamma$ et la thermogenèse adaptative (pour revue, voir [36]). Les résultats obtenus par I'utilisation de ligands de PPAR $\alpha$ et PPAR $\gamma$ en culture, ainsi que par leur administration à des souris, suggèrent également que ces deux récepteurs sont impliqués dans le contrôle positif de I'expression des UCP2 et UCP3 ([35], pour revue, voir [36]). PPAR $\alpha$ pourrait d'autre part être impliqué dans l'activité d'oxydation des acides gras dans le tissu adipeux brun, bien que les souris déficientes en PPAR $\alpha$ s'accommodent relativement bien $d^{\prime}$ 'une exposition au froid $[30,31]$.

Les fonctions respectives de ces UCP ne sont pas encore claires, et leur contribution au maintien du poids corporel et au développement de l'obésité est actuellement en cours d'investigation [37]. En effet, bien que la fonction du tissu adipeux brun humain soulève d'importantes controverses en dehors de la période néonatale, la participation de la thermogenèse adaptative au maintien de l'équilibre énergétique chez l'homme n'est pas totalement exclue, principalement en raison de l'expression de certaines UCP dans le muscle squelettique (pour revue, voir [36], [38]).

\section{Les bases de l'insulino- résistance induite par l'obésité}

L'insulino-résistance est un trouble métabolique complexe, due à un défaut d'efficacité de I'insuline. II en résulte une faible utilisation du glucose par les tissus périphériques (tissu adipeux, foie, muscle squelettique), et ce malgré la présence d'une quantité importante d'insuline dans la circulation sanguine. L'obésité d'une part, I'hyperlipidémie d'autre part, sont des facteurs prédisposants à cette affection, puisque l'obésité est présente dans plus de $80 \%$ des cas de diabète de type II. 
Le TNF $\alpha$ (tumor necrosis factor $\alpha$ ) produit par les adipocytes, pourrait être I'un des facteurs impliqués dans le développement de l'insulinorésistance. En dehors de sa participation aux défenses immunitaires en cas de tumeur ou d'infection, le TNF $\alpha$ est également sécrété par les adipocytes du tissu adipeux blanc. Tout comme la leptine, il stimule la lipolyse, et provoque par conséquent une augmentation de la quantité d'acides gras circulants qui entrent en compétition avec le glucose comme source d'énergie. De plus, le TNF $\alpha$ contrecarre l'action de l'insuline sur le muscle et le tissu adipeux blanc, en altérant la cascade de signaux induits sous son effet $[39,40]$. L'analyse de souris déficientes en TNF $\alpha\left(T N F \alpha^{-1}\right)$ montre qu'elles sont en effet protégées de l'insulinorésistance induite par l'obésité [41]. La cohérence de ces résultats est cependant mise en cause par le fait que, chez la souris, la neutralisation du récepteur au TNF $\alpha$ conduit à une hyperinsulinémie [42]. Par ailleurs, chez des rats âgés, il n'existe pas de lien entre I'augmentation de TNF $\alpha$ produit par le tissu adipeux et le développement d'une insulinorésistance [43], ce qui suggère l'existence de mécanismes d'insulinorésistance indépendants de la voie de signalisation du TNF $\alpha$.

Un autre mécanisme invoqué dans le diabète de type II est celui de I'accumulation de graisses dans les cellules musculaires parallèlement au développement de l'obésité. C'est le dysfonctionnement de ces cellules, grandes utilisatrices de glucose et $d^{\prime}$ acides gras, qui serait en partie responsable du syndrome d'insulinorésistance. Dans ce contexte, I'action hypoglycémiante et hypolipidémiante des TZD pourrait être due à l'activation de PPAR $\gamma$ dans les adipocytes blancs, améliorant ainsi la prise en charge des acides gras circulants par le tissu adipeux. L'effet obtenu est la baisse du taux d'acides gras circulants, la diminution de la charge en lipides des cellules musculaires et en conséquence, I'amélioration de l'utilisation du glucose par ces cellules [44]. Cependant, les TZD sont efficaces même chez des souris déficientes en tissu adipeux blanc, suggérant que PPAR $\gamma$ dans les adipocytes n'est pas leur unique cible [45].

Alors que I'activation de PPAR $\gamma$ amé- liore la sensibilité à l'insuline, une diminution de l'activité de ce récepteur pourrait prévenir l'apparition de l'obésité et de l'insulinorésistance qui lui est associée. En effet, des résultats observés chez des souris hétérozygotes pour le gène de PPAR $\gamma$ montrent que lorsque ces animaux sont soumis à un régime alimentaire riche en graisses, ils sont protégés à la fois contre une augmentation exagérée de leur masse adipeuse et contre l'apparition consécutive d'une résistance à I'insuline [26]. L'hypertrophie du tissu adipeux caractéristique de l'obésité, et conduisant à l'insulinorésistance nécessiterait les deux allèles du gène de PPAR $\gamma$.

Chez I'homme, I'existence de mutations dans le gène $P P A R \gamma$ est corrélée avec des variations dans la sensibilité à l'insuline, en association ou non à I'obésité (Tableau I). Une mutation ayant pour conséquence un gain d'activité de PPAR $\gamma$ a été identifiée chez trois patients souffrant à la fois d'obésité et de diabète insulinorésis-

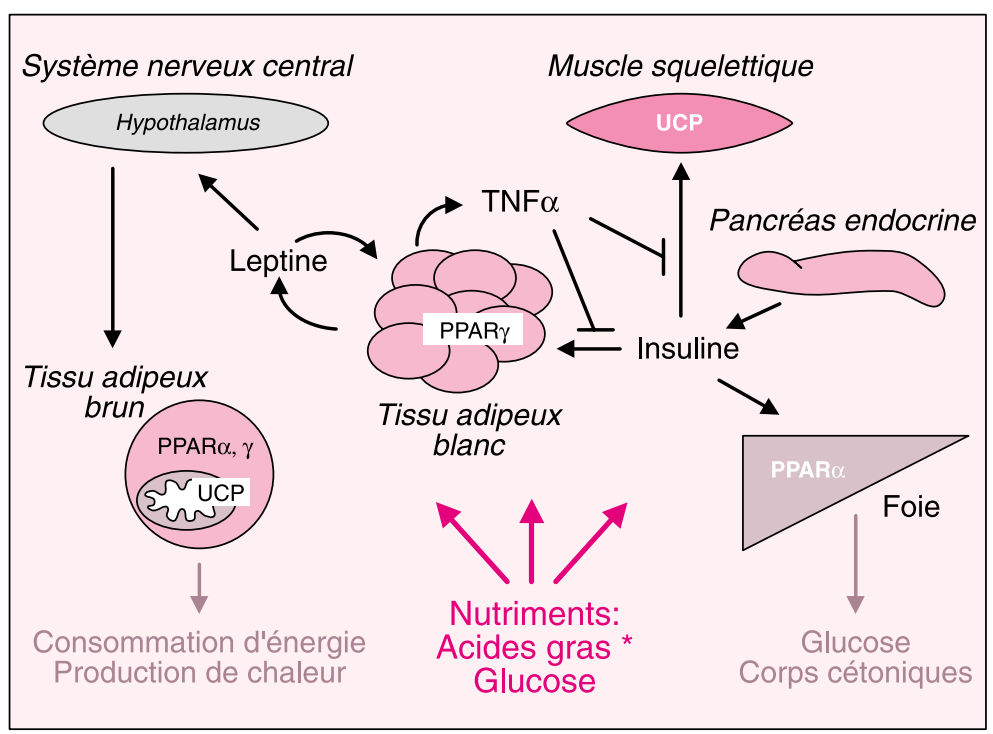

Figure 3. Principaux organes impliqués dans les interactions nécessaires au maintien de l'équilibre énergétique et du poids corporel. Les mécanismes de rétrocontrôle, les interactions entre les tissus au niveau périphérique, associées à l'intervention du système nerveux central, ainsi que les interactions entre le métabolisme des lipides et celui du glucose sont essentiels pour maintenir le contrôle du métabolisme énergétique et du poids corporel. L'obésité et l'hyperlipidémie sont des maladies multifactorielles, dont le développement résulte le plus souvent d'un dérèglement de plusieurs de ces voies de contrôle physiologiques. L'importance d'un terrain génétique prédisposant est maintenant reconnue, et certaines des mutations associées au développement de l'obésité chez l'homme altèrent le fonctionnement de molécules impliquées dans ces voies métaboliques. * : les acides gras peuvent être considérés comme des molécules de signal, agissant par l'intermédiaire des PPAR, dont ils contrôlent l'activité transcriptionnelle. 
de ces sources d'énergie (figure 3). Les interactions entre ces tissus sont en partie contrôlées par l'insuline. L'augmentation de la sécrétion d'insuline en réponse à une augmentation de la glycémie stimule le stockage du glucose tout en diminuant sa production dans le foie. Elle joue également un rôle important dans le métabolisme des lipides, puisqu'elle stimule le stockage des triglycérides dans le tissu adipeux blanc, ainsi que la synthèse des acides gras et des triglycérides en agissant sur des enzymes lipogéniques. De plus, I'insuline serait capable, non seulement d'augmenter l'expression de PPAR $\gamma$ dans le tissu adipeux blanc [47] mais aussi de stimuler I'action adipogénique de PPAR $\gamma$. Enfin, I'insuline exerce également un contrôle négatif sur l'expression de PPAR $\alpha$ dans le foie, et il apparaît clairement que les défauts du métabolisme des lipides et de celui du glucose vont de pair chez les souris déficientes en PPAR $\alpha[30,31]$.

\section{Conclusions}

Notre compréhension encore incomplète des mécanismes de contrôle de la balance énergétique nous permet de dégager quelques éléments clés. La leptine et les acides gras sont les molécules indicatrices de l'état énergétique de l'organisme, le système nerveux central est le principal lieu d'intégration de ces informations, et le tissu adipeux, le pancréas, le muscle squelettique et le foie sont les effecteurs qui agissent pour le maintien de I'équilibre énergétique. II apparaît clairement que ces effecteurs fonctionnent de manière concertée, en interagissant les uns avec les autres d'une façon étroitement contrôlée. La découverte des différentes voies métaboliques impliquées dans ces mécanismes complexes, ainsi que les résultats importants qui découlent de l'inactivation de l'un de ces acteurs sur le métabolisme général (modèles expérimentaux ou mutations) permettent $d^{\prime}$ insister sur trois considérations majeures.

La première est que les mécanismes de rétrocontrôle, $d^{\prime}$ 'interactions entre les tissus, d'interactions entre le métabolisme des lipides et celui du glucose se révèlent être précis et cruciaux, et permettent à la plupart des individus de maintenir un contrôle sur leur métabolisme énergétique et leur poids.

La deuxième est qu'il apparaît clairement que l'obésité et I'hyperlipidémie sont des maladies multifactorielles, dans lesquelles le fond génétique joue un rôle important, et ne sont pas la simple conséquence d'un régime alimentaire inadapté.

Enfin, la troisième conséquence est que de nombreuses cibles thérapeutiques potentielles s'offrent à nous. L'administration de leptine a déjà fait I'objet de tests cliniques, mais son intérêt pour le traitement de l'obésité reste limité puisque dans la majorité des cas, l'obésité est associée à une résistance plutôt qu'à un déficit en leptine [2]. Les UCP pourraient elles aussi se révéler des cibles thérapeutiques, s'il s'avère que les protéines de cette famille récemment identifiées (UCP2, UCP3, UCP4) jouent réellement un rôle dans le développement de l'obésité humaine. Dans ce cas, des molécules capables de les stimuler faciliteraient la dépense d'énergie et lutteraient ainsi contre I'obésité (pour revue, voir [33]). Cependant, en raison des controverses soulevées par la fonction du tissu adipeux brun chez l'homme adulte, cette voie thérapeutique paraît incertaine à I'heure actuelle. Enfin, nous avons vu que les PPAR sont des facteurs clés dans le maintien de l'équilibre énergétique. De plus, I'utilisation des fibrates (ligands de PPAR $\alpha$ ) comme hypolipidémiants, et des TZD (ligands de PPAR $\gamma$ ) comme hypoglycémiants, a déjà démontré que ces récepteurs nucléaires sont des cibles thérapeutiques d'un très grand intérêt. Des efforts importants sont actuellement entrepris dans le but d'identifier de nouvelles molécules, agonistes ou antagonistes des PPAR, pouvant être utilisées dans le traitement de I'hyperlipidémie et de I'obésité. On peut également imaginer que des co-facteurs impliqués dans I'activité de ces récepteurs nucléaires, tels que PGC-1 et PGC-2 pourraient dans l'avenir se révéler des cibles thérapeutiques. Toutefois, nous n'avons à l'heure actuelle aucune connaissance du type de molécules qui seraient capables de cibler ces cofacteurs.

Le nombre de cibles thérapeutiques potentielles augmente à mesure que progressent les connaissances des mécanismes moléculaires du développement de I'obésité. Cependant, l'obésité étant un syndrome complexe et multifactoriel, son traitement doit prendre en compte non seulement les réalités physiologiques liées au terrain génétique, mais également les facteurs comportementaux, nutritionnels et psychologiques

\section{Remerciements}

Nous remercions Jennifer Rieusset et Sander Kersten pour la lecture critique de ce manuscrit. Les travaux de recherche du laboratoire sont soutenus par le Fonds National Suisse pour la Recherche Scientifique, par I'Etat de Vaud et par le Human Frontier Science Program.

\section{RÉFÉRENCES}

En raison des contraintes concernant le nombre de références, toutes les contributions importantes dans les différents domaines abordés n'ont malheureusement pu être citées. Nous nous en excusons auprès des auteurs. Ces références sont citées dans les articles de revue auxquels nous renvoyons les lecteurs.

1. Schwartz MW, Woods SC, Porte D J, Seeley RJ, Baskin DG. Central nervous system control of food intake. Nature 2000;404: 661-71.

2. Friedman JM, Halaas JL. Leptin and the regulation of body weight in mammals. Nature 1998; 395: 763-70.

3. Zhang $Y Y$, Proenca $R$, Maffei $M$, Barone $M$, Leopold L, Friedman JM. Positional cloning of the mouse obese gene and its human homologue. Nature 1994; 372: 425-32.

4. Tartaglia LA, Dembski M, Weng X, et al. Identification and expression cloning of a leptin receptor, OB-R. Cell 1995; 83:126371

5. Montague CT, Faroogi IS, Whitehead JP, et al. Congenital leptin deficiency is associated with severe early-onset obesity in humans. Nature $1997 ; 387$ : 903-8.

6. Strobel A, Issad T, Camoin L, Ozata M, Strosberg AD. A leptin missense mutation associated with hypogonadism and morbid obesity [news]. Nat Genet 1998; 18: 213-5.

7. Clement K, Vaisse C, Lahlou N, et al. A mutation in the human leptin receptor gene causes obesity and pituitary dysfunction [see comments]. Nature 1998; 392: 398-401.

8. Rosenbaum M, Leibel RL. The role of leptin in human physiology [editorial; comment]. N Engl JMed 1999; 341: 913-5. 


\section{RÉFÉRENCES}

9. Kim I, Kim HG, Kim H, et al. Hepatic expression, synthesis and secretion of a novel fibrinogen/angiopoietin-related protein that prevents endothelial-cell apoptosis. Biochem J 2000; 346 Pt 3 : 603-10.

10. Yoon JC, Chickering TW, Rosen ED, et al. Peroxisome proliferator-activated receptor gamma target gene encoding a novel angiopoietin-related protein associated with adipose differentiation. Mol Cell Biol 2000 20: 5343-9.

11. Kersten S, Mandard S, Tan NS, et al. Characterization of the fasting induced adipose factor FIAF, a novel PPAR target gene. J Biol Chem 2000 (sous presse).

12. Desvergne B, Wahli W. Peroxisome proliferator-activated receptors: nuclear control of metabolism. Endocr Rev 1999; 20: 649. 88.

13. Nuclear Receptors Nomenclature Committee. A unified nomenclature system for the nuclear receptor superfamily. Cell 1999 . 97: 161-3.

14. Willson TM, Wahli W. Peroxisome proliferator-activated receptor agonists. [Review]. Curr Opin Chem Biol 1997; 1: 235-41.

15. Rosen ED, Sarraf P, Troy AE, et al. PPAR gamma is required for the differentiation of adipose tissue in vivo and in vitro. Mol Cell $1999 ; 4: 611-7$.

16. Bastie C, Holst D, Gaillard D, Jehl-Pietri C, Grimaldi PA. Expression of peroxisome proliferator-activated receptor PPARdelta promotes induction of PPARgamma and adipocyte differentiation in 3T3C2 fibroblasts. Biol Chem 1999; 274: 21920-5.

17. Tontonoz P, Hu E, Graves RA, Budavar AI, Spiegelman BM. MPPAR gamma 2 : tissue-specific regulator of an adipocyte enhancer. Genes Dev 1994; 8: 1224-34.

18. Castillo G, Brun RP, Rosenfield JK, et al. An adipogenic cofactor bound by the differentiation domain of PPARgamma. EMBO $1999 ; 18: 3676-87$.

19. Pearson SL, Cawthorne MA, Clapham IC, et al. The thiazolidinedione insulin sensitiser, BRL 49653, increases the expression of PPAR-gamma and aP2 in adipose tissue of high-fat-fed rats. Biochem Biophys Res Commun 1996 ; 229: 752-7.

20. Vidal-Puig A, Jimenez-Linan M, Lowell $\mathrm{BB}$, et al. Regulation of PPAR gamma gene expression by nutrition and obesity in rodents. J Clin Invest 1996; 97 : 2553-61.

21. Shimoike $T$, Yanase $T$, Umeda $F$, Ichino I, Takayanagi R, Nawata H. Subcutaneous or visceral adipose tissue expression of the PPARgamma gene is not altered in the fatty $(\mathrm{fa} / \mathrm{fa})$ Zucker rat. Metabolism 1998; 47: 1494-8.

22. Vidal-Puig AJ, Considine RV, JimenezLinan $M$, et al. Peroxisome proliferator-activated receptor gene expression in human tissues. Effects of obesity, weight loss, and regulation by insulin and glucocorticoids. J Clin Invest 1997; 99: 2416-22.

23. Ristow M, Müller-Wieland D, Pfeiffer A, Krone W, Kahn CR. Obesity associated with a mutation in a genetic regulator of adipocyte differentiation. $N$ Engl J Med 1998; 339 : 953-9.

24. Deeb SS, Fajas L, Nemoto $M$, et al. A Pro12Ala substitution in PPARgamma2 associated with decreased receptor activity, lower body mass index and improved insulin sensitivity. Nat Genet 1998; 20: 284-7.

25. De Vos P, Lefebvre AM, Miller SG, et al. Thiazolidinediones repress ob gene expression in rodents via activation of peroxisome proliferator-activated receptor gamma. J Clin Invest 1996; 98 : 1004-9.

26. Kubota $\mathrm{N}$, Terauchi $\mathrm{Y}$, Miki $\mathrm{H}$ et al. PPAR gamma mediates high-fat diet-induced adipocyte hypertrophy and insulin resistance. Mol Cell 1999; 4: 597-609.

27. Kim JB, Sarraf $P$, Wright $M$, et al. Nutritional and insulin regulation of fatty acid synthetase and leptin gene expression through ADD1/SREBP1. J Clin Invest 1998. 101: 1-9.

28. Amri EZ, Bonino F, Ailhaud G, Abumrad NA, Grimaldi PA. Cloning of a protein that mediates transcriptional effects of fatty acids in preadipocytes. Homology to peroxisome proliferator-activated receptors. J Biol Chem 1995; 270: 2367-71.

29. Peters JM, Lee SS, Li W, Ward JM, et al. Growth, adipose, brain, and skin alterations resulting from targeted disruption of the mouse peroxisome proliferator-activated receptor beta(delta). Mol Cell Biol 2000 . $20: 5119-28$

30. Kersten S, Seydoux J Peters IM, Gonzalez FI, Desvergne B, Wahli W. Peroxisome proliferator-activated receptor alpha mediates the adaptive response to fasting. Clin Invest 1999; 103: 1489-98.

31. Leone TC, Weinheimer CJ, Kelly DP. A critical role for the peroxisome proliferatoractivated receptor alpha (PPARalpha) in the cellular fasting response: the PPARalphanull mouse as a model of fatty acid oxidation disorders. Proc Natl Acad Sci USA $1999 ; 96: 7473-8$

32. Enerback S, Jacobsson A, Simpson EM, et al. Mice lacking mitochondrial uncoupling protein are cold-sensitive but not obese. Nature 1997 ; 387 : 90-4.

33. Ricquier D, Bouillaud F. Les protéines découplantes mitochondriales. Med SCi 1998; 14:889-97.

34. Mao W, Yu XX, Zhong A, et al. UCP4, a novel brain-specific mitochondrial protein that reduces membrane potential in mammalian cells [published erratum appears in FEBS Lett 1999; 449: 293]. FEBS Lett 1999; 443: $326-30$

35. Kelly LJ, Vicario PP, Thompson GM, et al. Peroxisome proliferator-activated receptors gamma and alpha mediate in vivo regulation of uncoupling protein (UCP-1, UCP-2,
UCP-3) gene expression. Endocrinology $1998 ; 139$ : 4920-7.

36. Lowell BB, Spiegelman BM. Towards a molecular understanding of adaptive thermogenesis. Nature 2000; 404: 652-60.

37. Ricquier D. Uncoupling protein-2 (UCP2): molecular and genetic studies. Int I Obes Relat Metab Disord 1999; 23 Suppl 6: S38-42.

38. Hirsch J. Obesity. Some heat but not enough light. Nature 1997 ; 387 : 27-8.

39. Hotamisligil GS, Peraldi P, Budavari A, Ellis R, White MF, Spiegelman BM. IRS-1mediated inhibition of insulin receptor tyrosine kinase activity in TNF-alpha- and obesity-induced insulin resistance. Science $1996 ; 271$ : 665-8.

40. Valverde AM, Teruel T, Navarro $P$, Benito $M$, Lorenzo $M$. Tumor necrosis factor-alpha causes insulin receptor substrate2- mediated insulin resistance and inhibits insulin-induced adipogenesis in fetal brown adipocytes. Endocrinology 1998; 139: 1229-38.

41. Uysal KT, Wiesbrock SM, Marino MW, Hotamisligil GS. Protection from obesityinduced insulin resistance in mice lacking TNF- alpha function. Nature 1997; 389: 610-4.

42. Schreyer SA, Chua SC Ir, LeBoeuf RC. Obesity and diabetes in TNF-alpha receptordeficient mice. J Clin Invest 1998; 102 : 402-11.

43. Morin CL, Gayles EC, Podolin DA, Wei $\mathrm{Y}, \mathrm{Xu}$ M, Pagliassotti MJ. Adipose tissuederived tumor necrosis factor activity correlates with fat cell size but not insulin action in aging rats. Endocrinology 1998; 139: 4998-5005.

44. Martin G, Schoonjans K, Staels B, Auwerx J. PPARgamma activators improve glucose homeostasis by stimulating fatty acid uptake in the adipocytes. Atherosclerosis 1998; 137 Suppl: S75-80.

45. Burant CF, Sreenan S, Hirano KI, et al. Troglitazone action is independent of adipose tissue. J Clin Invest 1997; 100: 29008.

46. Barroso I, Gurnell M, Crowley VE, et al. Dominant negative mutations in human PPARgamma associated with severe insulin resistance, diabetes mellitus and hypertension Nature $1999 \cdot 402: 880-3$

47. Rieusset J, Andreelli F, Auboeuf D, et al. Insulin acutely regulates the expression of the peroxisome proliferator- activated receptor-gamma in human adipocytes. Diabetes $1999 ; 48$ : 699-705.

\section{TIRÉS À PART}

W. Wahli. 
$-\mathbf{m} / \mathbf{S} 2000$

\section{Summary}

Obesity: molecular basis

and therapeutic targets

Obesity is an increasingly serious health problem, and is highly associated with insulin-resistance and dyslipidemia. The mechanisms involved in the development of this disorder are still poorly understood, although significant progress has been recently made in the elucidation of their molecular basis. The major causes leading to obesity are defects in the regulation of fat metabolism. Several mutations identified in different animal models have unveiled the roles of a number of genes in the regulation of energy balance. These dicoveries, together with the fact that some of these mutations have been found in humans, have lead to the conclusion that obesity is due to nutritional or environmental factors, but also involves genetic factors. A number of important peripheric factors participate in the regulation processes, such as the adipocyte-specific hormone leptin, and the nuclear homone receptors PPARs. A general scheme can now be drawn which includes some key factors and their respective interactions.

\section{taf Club Francophone des Cellules Dendritiques Site Internet : http://www.cochin.inserm.fr/CFCD}

ATELIER

\section{Infection transmuqueuse des Cellules Dendritiques}

Organisation: Daniel Bout, Colette Dezutter-Dambuyant, Anne Hosmalin, Laurent Misery

Lundi 27 novembre 2000

Hôpital Cochin, ICGM, $2^{\mathrm{e}}$ étage, 22, rue Méchain, 75014 Paris

13 h 00 à 17 h 30 - limité à 80 participants

Programme:

- Physiologie des cellules dendritiques dans les différents types de muqueuses

- Micro-organismes et muqueuses : place des cellules dendritiques

- Modèles in vivo d'infection de cellules dendritiques des muqueuses

- Modèles in vitro d'infection de cellules dendritiques des muqueuses

- Table ronde: Entrée des micro-organismes, validité des modèles, induction de l'immunité muqueuse,

tests de contrôle de l'immunité muqueuse, perspectives de nouvelles voies vaccinales

\section{CONGRÈS ANNUEL DU CFCD \\ Mardi 28 novembre 2000 \\ Maison de l'UNESCO, 7, place Fontenoy, 75007 Paris}

Le Congrès inclut des conférences, des communications orales et communications affichées.

* Session du matin : 28 novembre 2000 (9 h 30-12 h 15)

$>$ Conférence: Philippe Pierre (France)

Biologie cellulaire de la présentation de l'antigène classe II restreinte dans les Cellules Dendritiques Communications libres

$>$ Conférence: Cornelis Melief (Pays-Bas)

Anti-tumor immunotherapy - Immunothérapie antitumorale

* Assemblée Générale du CFCD

* Déjeuner-Buffet avec visite des communications affichées (posters)

* Session de l'après-midi : 28 novembre 2000 (15 h 15-18 h 30) Communications libres

$>$ Conférence spéciale: Jacques Banchereau (USA)

Sous-populations de Cellules Dendritiques et leur utilisation

Contact SECRÉtariat CFCD : Colette Dezutter-Dambuyant

Unité Inserm U. 346, Hôpital Édouard-Herriot

69437 LYON Cedex 03

e-mail : dezutter@lyon151.inserm.fr 